\title{
Standard Model Review and a New 5D Multi-Brane Proposition
}

\author{
Jami Hossain \\ Independent Researcher, Gurgaon, India \\ Email: hossainjami@yahoo.com
}

How to cite this paper: Hossain, J. (2022) Standard Model Review and a New 5D Multi-Brane Proposition. Journal of High Energy Physics, Gravitation and Cosmology, 8, 195-227.

https://doi.org/10.4236/jhepgc.2022.81015

Received: November 30, 2021

Accepted: January 21, 2022

Published: January 24, 2022

Copyright $\odot 2022$ by author(s) and Scientific Research Publishing Inc. This work is licensed under the Creative Commons Attribution International License (CC BY 4.0).

http://creativecommons.org/licenses/by/4.0/

\begin{abstract}
Based on a comprehensive review of mainly the non-quantum aspects of the standard model of cosmology, the 5 dimensional models, and the analysis here, we propose a 5 dimensional model with expanding $4 \mathrm{D}$ multi-branes. A review of the standard model in the context of many new developments and discoveries in cosmology in the recent times, such as the accelerated expansion of the universe, Plank cosmic microwave measurements, dark energy survey, Hubble tension etc. tends to indicate that the standard model is essentially a patchwork of different theoretical models that have been pieced together in an attempt to explain different aspects of the astrophysical observations, which do not necessarily emanate from a full end-to-end understanding of a physical process. The purpose of each individual theoretical piece such as "inflation" is limited to providing an explanation to the problem area or a gap in our understanding. A number of new theories such as the fivedimensional universe, the bulk and brane, extended theories of gravity, and conformal cyclic cosmology offer alternate ways of addressing the existential aspects of the universe but these models too remain hypothetical with shortcomings and a lack of conclusive evidence. The model proposed by us, presents a way forward in addressing dark matter and dark energy as manifestations of the multiple underlying branes in the aftermath of the big-bang. In the process, we present a theorem of the dimensionality of the expanding universe, which necessitates the need for at least one more dimension in addition to the 4 dimensions of spacetime. While carrying out the review of the standard model, we present new analysis and facts that strengthen the case for the $5^{\text {th }}$ dimension. According to the multi-brane hypothesis presented here, our observed universe could be one of the many branes, and it is more likely than not that in the aftermath of the big-bang that generated our brane, more branes were generated, which further points towards a much more prolonged big-bang event than what has been the perception so far.
\end{abstract}


Keywords

Big-Bang, Dark Matter, Dark Energy, Cosmological Constant, Standard Model, Brane, 5D Universe, Accelerated Expansion

\section{Introduction}

We begin with this relevant quote by Roger Penrose:

"My arguments do not depend on detailed calculations, but on what seem to me to be certain 'obvious' facts, whose very obviousness may contribute to their being frequently overlooked."

Roger Penrose

Recent problems and discoveries in cosmology such as the Hubble tension [1] [2] [3] [4] [5], the giant arc [6] [7] [8] [9] accelerated expansion of the universe [10], inflation [11], and the yet to be conclusively established dark energy and cold dark matter (CDM) [12] in the $\Lambda$ CDM model are raising some doubts and questions [13] on the standard model of cosmology (SMC) [14] [15] [16]. Over the last few decades, research in cosmology has become highly focused and specialized in niche areas and while there have been many breakthroughs, less work has been carried out to holistically view the developments and to connect the dots. A high level of mathematical and conceptual complexity in diverse areas such as the quantum field theory (QFT) [17] on the one hand and "inflation" and Conformal Cyclic Cosmology (CCC) [18] on the other hand, makes the task of a comprehensive review of the SMC even more challenging. This paper recognizes many lacunae in our understanding of the evolution of the universe and makes a modest attempt to carry out a review of mainly the non-quantum aspects of SMC from a view point of identifying the gaps in our understanding. Discussion and analysis carried out here show that in addition to many of the well established assumptions (or hypotheses) such as inflation, dark energy, and dark matter, there can be other alternatives to the evolutionary path and dimensionality of the universe, which can be considered on similar levels of confidence. These other alternatives, mainly revolving around a 5 dimensional hypothesis, are also reviewed. Though $5 \mathrm{D}$ bulk and brane models have been proposed earlier by Randall and Sundrum (1999) [19], Kaluza (1921) [20], Wesson (2012) [21], they have been lacking in an evidence of the $5^{\text {th }}$ dimension. That Einstein's theory of gravity may not be an entirely definitive theory, also maintained by many, has led to "extended theories of gravity (ETG)" [22] [23] [24] which explore alternatives and explanations other than the standard picture. In this paper, in addition to the review of SMC, extended theories of gravity and 5D hypotheses, we delve deeper into understanding of time and dimensionality to present a general theorem of the dimensionality of the expanding universe, which further strengthens the case for the $5^{\text {th }}$ dimension (5D) and a multibrane expanding universe with $4 \mathrm{D}$ branes and a 5D bulk. The implications of a 5D big bang and 
its aftermath on the cosmological constant $\Lambda$, dark matter and dark energy are discussed.

\subsection{Anomalies and Lacunae in SMC}

SMC can be regarded as a consensus theory broadly accepted in the scientific community. It rests on a massive body of work carried out over the last century with key elements of modern physics plugging-in to provide us with a model of the evolution of the universe. General Theory of Relativity (GTR) [25] [26] [27] [28] [29], is the underlying foundational model of the SMC.

The cosmological constant $\Lambda$ [30], which is the most important parameter in SMC, and determines how the universe will evolve and perhaps also how it will end, has been a subject of intense scientific scrutiny over the last 100 years. Now its purpose is somewhat different from the original intent, of keeping the universe stationary in the Einstein's original model (See Section 2.0 for equations). It is the term that allows for vacuum energy and the accelerated expansion of the universe [31] [32]. Adam Riess et al. (1998) [10] based on SN Ia spectroscopic analysis conclude accelerated expansion of the universe. $\Lambda$ also happens to be the bridge between the cosmological model and the quantum world, related in part to stress-energy density of the vacuum [33].

In connection with the cosmological constant $\Lambda$, Perlmutter et al. (1999) [34] working on probability distribution of cosmological parameters, conclude that the data are strongly inconsistent with $\Lambda=0$, flat or open cosmology. On the other hand the data indicates with a confidence level of $99 \%$ that $\Lambda \neq 0$ and is positive. Commenting on cosmological implications of this work, they have identified two cosmological coincidences that need to be explained 1) very small but non-zero energy density and 2) closeness of the cosmological constant value to mass-energy density. In relationship (15) of section 2.0, we note that $\Omega_{m}$ and $\Omega_{\Lambda}$ should diverge rapidly from their initial conditions with the evolution of the universe. Therefore, according to Perlmutter, one would require initial conditions in which the ratio of densities is of the order of $10^{-100}$ for the two densities to coincide today. They also conjecture on the possibility of a previously unknown physical entity that contributes to the universe's total energy density and results in different expansion history than with the cosmological constant. This thought by Perlmutter of the "unknown physical entity" is an important indicator of certain shortcomings and inadequacy of SMC in fully describing the evolution of the universe.

Independent methods to estimate Hubble's constant have not only yielded different values but also different levels of corresponding uncertainties and somewhat low confidence levels $\sim 68 \%$. Prominent amongst them is the Planck Legacy [35] measurements of Cosmic Microwave Background (CMB) anisotropies [36] with an estimate of about $67 \mathrm{~km}^{-1} \cdot \mathrm{Mpc}^{-1}$ and the Hubble Space Telescope (HST) GAIA EDR3 method estimate at around $73-74 \mathrm{~km}^{-1} \cdot \mathrm{Mpc}^{-1}$ [37]. Valentino et al. [38] warn of profound implications to the $\Lambda \mathrm{CDM}$ model based on lensing anomaly in the Plank Legacy18 observations of CMB spectra. Lensing anomaly re- 
lates to the fact that the latest analysis of CMB anisotropies by Planck 2018 has found that there is $10 \%$ more lensing than expected in the CMB power spectrum [39].

The Dark Energy Survey Collaboration (DES) in creating a detailed map of the dark matter distribution in the universe claims a deviation from the Theory of Relativity (TR) [40]. Blandford et al. [41] comment:

"cosmologists must also confess their ignorance. They do not know the identity of dark matter, they cannot explain why a tiny fraction of baryons should survive the early universe, they do not understand the mechanics of inflation and they cannot account for the cosmological constant."

On the other hand, the giant arc [42] [43] located 9.2 billion light years away and 3.3 billion light years across, which along with some of the other large structures such as Sloan Great Wall, the Huge Large Quasar Group, and the Hercules-Corona Borealis Great Wall [44] seems to present a case of a departure from homogeneity and isotropy assumptions [45] in $\Lambda$ CDM model.

LHCb Collaboration (March 2021) [46] have found that B + mesons decay to muons about $25 \%$ less often than they decay to electrons, hinting at the possibility of the violation of lepton universality ${ }^{1}$. The dominance of matter over antimatter and the apparent dark-matter content of the universe or the baryon asymmetry of the universe (BAU), cannot be explained in the SMC [47]. The breakup of matter-energy in the universe is $-68 \%$ dark energy, $27 \%$ dark matter, and 5\% matter [48], which means that SMC relies on an assumption of $95 \%$ of energy and force of an unknown type and emanating from an unknown source. This fact weakens SMC quite significantly.

In brief, the SMC is as follows:

The universe in current time has evolved from a highly miniscule region of space or a singularity, due to an event known as the "big bang" and a subsequent expansion [11] [12] [18]. Here there are two alternate theories-1) that of a big bang event at around $10^{-45} \mathrm{~s}$, when the universe is of the size of a few $\mathrm{mm}$ and that point in time, the expansion is almost linear; and 2) that of exponential expansion (inflation) between $10^{-36} \mathrm{~s}$ and $10^{-32} \mathrm{~s}$ [49] [50] when the universe underwent a process of an incredible ultra inflation [11] [51], from a very hot and very high density state, assumed to be confined to a $4 \mathrm{D}$ hyper sphere in a micro space-time, increasing in physical size from almost $10^{-48} \mathrm{~cm}$, by an order of around $10^{27}$. Thereafter, i.e., $10^{-32} \mathrm{~s}$ onwards, both the theories predict almost linear expansion for the rest of the 13.77 billion years.

The SMC evolutionary time line in Figure 1 shows different phases of universe from the big bang some 13.77 billion years ago.

The CMB Thermal spectrum and the baryogenesis and nucleosynthesis [52] assessments for the early universe are seemingly well explained by the big-bang theory. The current Large Scale Structures (LSS) [53] that we see, are supposed

${ }^{1}$ Lepton universality is an aspect of SMPP, which means different leptons, electron $(\mathrm{e}-)$, muon $(\mu-)$ and tau $(\tau-)$, have the same interaction strengths. 


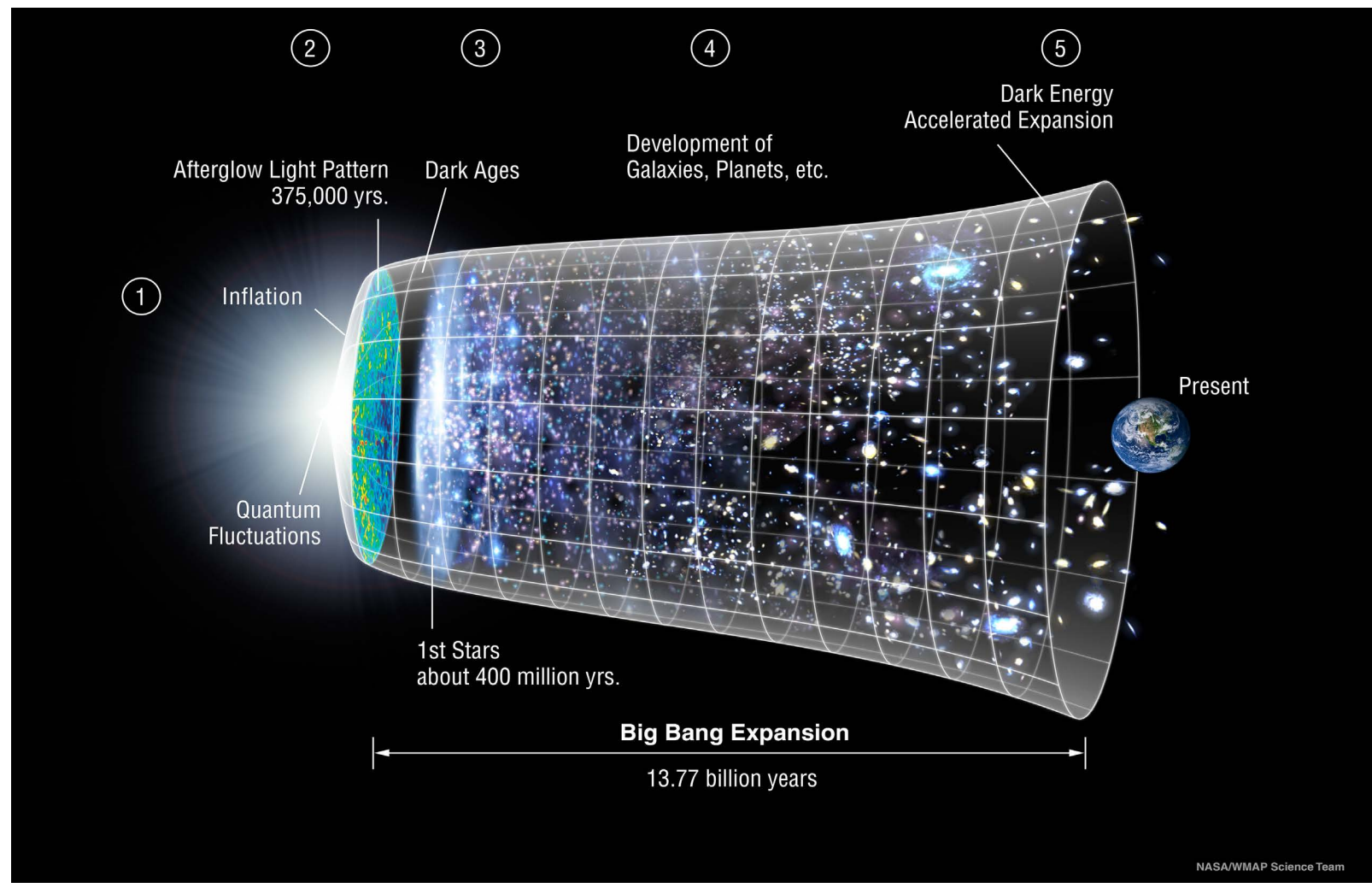

Figure 1. Evolutionary timeline of the Universe. Image Credit: NASA/LAMBDA Archive/WMAP science team.

to have evolved from the primordial perturbations or ripples in matter/energy density.

Theoretically, based on Friedmann-Robertson-Walker (FRW) equations, in the beginning there should be a cosmological singularity (CS) [54] [55] [56] [57]. The primordial inflationary epoch seems to have existed in the period $10^{-36}$ $10^{-32} \mathrm{~s}$

With regard to inflation, opinions differ and there are mainly two schools of thought, which we refer to as 1) the Guth School and 2) the Penrose School. The two schools of thought are briefly described below:

\subsubsection{Guth School}

According to Guth (2002) [11], the universe begins at a size of around $10^{-48} \mathrm{~cm}$, a hundred billion times smaller than a proton and at $10^{-44} \mathrm{~s}$ (Planck time) from the "big bang". Before $10^{-44} \mathrm{~s}$, the universe should have been in a state of pure energy, when the temperature was of the order of $10^{32} \mathrm{~K}$ (Plank temperature). During inflation $\left(10^{-36}-10^{-32} \mathrm{~s}\right)$, the expansion was so ultrafast that energy and information would have to travel at $90-100$ times the speed of light in order to achieve homogeneity we see at around 375,000 years after the big bang in the form of CMB. This cannot be explained in the traditional big bang theory (without inflation). Guth turns to the concept of false vacuum, which is a negative pressure on a time scale of $10^{-37} \mathrm{~s}$, resulting in a repulsive force that drives the inflation. In the aftermath of inflation, there is a decay of false vacuum and 
that point onwards the inflationary and the traditional big bang theories follow the same evolutionary path.

\subsubsection{Penrose School}

Roger Penrose, on the other hand, is of the opinion that the concept of inflation is somewhat paradoxical, which he tries to explain in his Nobel lecture [58] and also in many other papers. According to him, CMB indicates a high entropy state but by current day physics, going back in time one would expect a low entropy state. He seems to be not convinced of the inflationary model, which is part of the SMC. A proposition made by Roger is that of Conformal Cyclic Cosmology (CCC), under which an initial space-time singularity can always be represented as a smooth past boundary to the conformal geometry of space-time. He equates singularity like high temperature conditions in distant past (13.77 billion years) to a distant future when the universe is completely cooled down and again there is no mass. According to him, absence of mass implies that the universe cannot keep track of time because the only other component left is photon, for which time doesn't elapse. We quote Roger where he summarizes his idea,

"With conformal invariance both in the remote future and at the Big-Bang origin, we can try to argue that the two situations are physically identical, so the remote future of one phase of the universe becomes the Big Bang of the next. This suggestion is my'outrageous' conformal cyclic cosmology (CCC)"

\subsubsection{Comments and Analysis}

Roger seems to negate inflation but not the big bang. It can be seen that the crux of the problem is high homogeneity or the high entropy of CMB. And a possible fix is "inflation" and the other one "CCC", both being patchwork in SMC to address homogeneity in the $\mathrm{CMB}$ afterglow.

Going by our current state of knowledge, inflation appears to be a logical and theoretical necessity as we work backwards towards the big-bang but Guth's inflationary model, which balances positive energy of the expansion with the creation of negative energy of gravity is challenging to the common sense as the universe practically becomes a free lunch and any sub-atomic particle anywhere can branch out as a universe by itself. How and why is not known. Guth admits that inflation, though it provides an explanation is not a proven theory.

Roger's CCC is mathematically and aesthetically appealing, but how does it reflect the physical reality of the universe? The concept of universe losing track of time or sort of "forgetting time" in a massless eon is a bit difficult to accept. Moreover, treating the two situations, one an infinitely expanded cold universe and the other, hot singularity as identical, is at least as challenging to the common sense as Guth's inflation. Why and how come a cold expanded universe, again converts into a superhot singularity? The physicality of the two situations defeats imagination.

Apart from many problem areas highlighted above, there are other questions-What was happening, say-between $0 \mathrm{~s}$ or $10^{-44} \mathrm{~s}$ till $10^{-36} \mathrm{~s}$ when the initiation of the inflation took place remains unexplained? What triggered infla- 
tion? In the neighborhood of $0 \mathrm{~s}$, when there is neither time nor space and the universe is presumably in an ideal and absolute equilibrium, what is it that triggered, first the big bang and then the inflation? It is also important to keep in mind that a "second" in the neighborhood of the CS where the density is around $10^{90} \mathrm{~kg} / \mathrm{cm}^{3}$, should by GTR, be a highly elongated "second" or in other words time was almost standstill. What then triggered a faster time lapse?

Be it CCC or inflation, questions rather difficult to address, nevertheless remain and cannot be swept aside. The above discussion is indicative of the fact that all is not well explained in the SMC. These shortcomings are not trivial and in recent times there has been a rising clamor for a new physics [59] [60].

There could be other possibilities which are not considered in the SMC. One of them is a positive influx of real energy into this universe, which is something that cannot be ruled out (discussed in section 6). Alternatively, it may also be possible that considering a $5 \mathrm{D}$ big bang offers a better solution. It is important to keep in mind that these and other possibilities that we discuss in this paper, though not without their own limitations of "how" and "why", can be considered at least with the same level of confidence as the SMC and the models proposed by Guth (inflationary model) and Roger (CCC).

Hossain (2019) [61] lists a number of fundamental concepts in physics on which we lack understanding about their true form e.g., mass, charge, and energy-though in recent times, attempts have been made to link such parameters to the geometry and dimensions of the space through string theory [62] [63], QFT [64], and bulk and brane theory [65].

Interestingly, so far, imaginative abstraction and theoretical work, to a certain extent is reasonably well matched with real world machines and experiments such as the Hubble Telescope [66] and the Large Hadron Collider (LHC) [67], which not only discovered the Higgs boson but also 62 new hadrons [68].

The most important aspects of SMC are dark matter and dark energy, which have been conceived as an explanation for the forces that keep the universe in an accelerated expansion mode. In that sense, these are essentially force equivalents that need not necessarily be in the undetectable matter and energy form as assumed in SMC. The force or forces could also be manifestations of a 5D Bulk on a $4 \mathrm{D}$ brane (discussed in later sections). Corda [24] have proposed detection of gravitational waves or perturbations other than those from Einstein's gravitational theory using Laser Interferometer Gravitational-Wave Observatory (LIGO) [69].

In Table 1, we summarize the main but largely non-quantum problem areas in SMC that need a resolution. It can be seen that SMC will need modifications, more evidence, and theoretical work in diverse areas to be acceptable as a complete theory of the evolution of the universe.

\section{Standard Model Description}

The two main components of SMC are 1) Standard Model of Particle Physics (SMPP) and 2) the General Theory of Relativity (GTR). 
Table 1. Some of the problem areas in SMC.

\begin{tabular}{|c|c|}
\hline Problem Area & Remarks \\
\hline Hubble Tension & $\begin{array}{l}4 \sigma-6 \sigma \text { Possible Modeling or } \\
\text { Systematic Error or failure in Lambda CDM, } \\
\text { Possible error in Standardising candles }\end{array}$ \\
\hline Lensing Anomaly & $\begin{array}{l}\text { Possible Systematic Error/Positive } \\
\text { Curvature/Closed Universe }\end{array}$ \\
\hline Accelerated Expansion & $\begin{array}{c}\text { Astrophysical Observations/Possibly New } \\
\text { Explanations }\end{array}$ \\
\hline $\mathrm{CDM}$ & Theoretical necessity (CMB observations) \\
\hline Dark Matter & Theoretical necessity/Astrophysical Observations \\
\hline Dark Energy & Theoretical Necessity/Astrophysical Observations \\
\hline Inflation & $\begin{array}{l}\text { Patchwork to SMC based on CMB and } \\
\text { Astrophysical Observations/Debatable. } \\
\text { Needs explanations and more evidence }\end{array}$ \\
\hline Conformal Cyclic Cosmology & $\begin{array}{c}\text { Alternative to inflation/Mathematically } \\
\text { and aesthetically appealing/Needs more } \\
\text { evidence and theoretical work }\end{array}$ \\
\hline Giant Arc & New Explanations needed \\
\hline Lepton Universality Violation & New Explanations needed \\
\hline Energy Density $\sim 0(>0)$ & New Explanations needed \\
\hline Critical Mass Density $(\sim 1)$ & New Explanations needed \\
\hline
\end{tabular}

In SMC, Einstein's General Theory of Relativity (GTR) [70] [71] forms the main underlying basis of the four dimensional $\Lambda \mathrm{CDM} \mathrm{model}^{2}$ given by:

$$
G^{\mu v}=\frac{8 \pi G}{c^{2}} T^{\mu v}-\Lambda g^{\mu v}
$$

where:

$G^{u v}$ is the Einstein Tensor, $T^{\mu v}$ is the Energy Momentum Stress Tensor, $g^{\mu v}$ is the metric tensor, and $\Lambda$ is the cosmological constant.

There are no solutions to (1) for a static, homogeneous universe, if the energy sources are in the form of matter and radiation (Carrol [31]). The work of Friedmann, Robertson and Walker (FRW) [72] established that the only homogeneous and isotropic geometries possible were with FRW equations given below in spherical coordinates $(r, \theta, \varphi)$ :

$$
\mathrm{d} s^{2}=\mathrm{d} r^{2}+f K(r)^{2} \mathrm{~d} \omega^{2}
$$

where:

$$
\mathrm{d} \omega^{2}=\mathrm{d} \theta^{2}+\sin ^{2} \theta \mathrm{d} \phi^{2}
$$

and $f K(r)$ is the curvature function defined as shown in Table 2.

${ }^{2}$ The underlying principles of GTR have been described in a number of textbooks and online resources, some of which are cited in this paper. 
Table 2. Curvature Function $f \mathrm{~K}(\mathrm{r})$.

\begin{tabular}{cccc}
\hline Curvatutre Function & $K>0$ & $K=0$ & $K<0$ \\
\hline$f k(r)$ & $K^{-\frac{1}{2}} \sin \left(K^{\frac{1}{2}} r\right)$ & $r$ & $K^{-\frac{1}{2}} \sinh \left(K^{\frac{1}{2}} r\right)$
\end{tabular}

And $K$ is the curvature constant defined by

$$
\text { Rcurve }=K^{-1 / 2}
$$

which in the observed universe is the Radius of a hypothetical $4 \mathrm{D}$ hypersphere (the expanding universe). In this, for $K \sim 0$, the $4 \mathrm{D}$ hyper-surface is flat and is called the Einstein de-Sitter static model, when $k=+1$ and $k=-1$ the four-space are of positive and negative constant curvature;

Equation (2) can also be written as:

$$
\mathrm{d} s^{2}=\frac{\mathrm{d} r^{2}}{1-K r^{2}}+r^{2} \mathrm{~d} \omega^{2}
$$

If we introduce time and a scale factor in Equation (2), we have

$$
\mathrm{d} s^{2}=-\mathrm{d} t^{2}+a(t)^{2}\left[\mathrm{~d} x^{2}+f K(x)^{2} x^{2} \mathrm{~d} \omega^{2}\right]
$$

where the function $a(t)$ is the time dependent scale factor, and the $r$ is replaced by $x$ representing the comoving radius of the sphere,

$$
r(t)=a(t) x
$$

$a(t)$ is normalized so that at present $a=1$ and $r=x$.

On inserting Equation (6) in Einstein's Equation (1), we get two of the equations, which represent diagonal components $(0,0)$ and $(i, i)$ :

$$
\begin{aligned}
& \left(\frac{\dot{a}}{a}\right)^{2}=\frac{8 \pi G}{3} \rho-\frac{K c^{2}}{a^{2}}+\frac{\Lambda}{3} \\
& \frac{\ddot{a}}{a}=-\frac{4 \pi G}{3}\left(\rho+\frac{3 p}{c^{2}}\right)+\frac{\Lambda}{3}
\end{aligned}
$$

These are the Friedmann Equations and can be combined to yield:

$$
\frac{\mathrm{d}}{\mathrm{d} t}\left(\rho a^{3} c^{2}\right)+P \frac{\mathrm{d}}{\mathrm{d} t}\left(a^{3}\right)=0
$$

Equation (7) can be written in the form:

$$
H^{2}=\frac{8 \pi G}{3}\left(\rho_{m}+\rho_{r}+\rho_{\Lambda}\right)-\frac{K c^{2}}{a^{2}}
$$

where $H$ is the Hubble's constant, and $\rho_{m}$ is the matter density from baryonic and cold dark matter, $\rho_{r}$ is the density corresponding to photons and neutrinos (massless particles), and $\rho_{\Lambda}=\frac{\Lambda}{8 \pi G}$, also related to the concept of vacuum energy. According to Carrol [31], vacuum energy density is associated with isotropic pressure. However, there is a major discrepancy between measured and theoretically computed values of $\rho_{\Lambda}$. This happens to be one of the problem areas in SMC. 
If we define critical density as

$$
\rho_{\text {crit }}=\frac{3 H^{2}}{8 \pi G}
$$

and set

$$
\rho_{\text {crit }}=\rho_{m}+\rho_{r}+\rho_{\Lambda}
$$

we get $K=0$. i.e., at critical density, the universe is flat.

In terms of dimensionless densities,

Friedmann equation can be written in terms of dimensionless densities as :

$$
H^{2}=H_{0}^{2}\left(\frac{\Omega_{m, 0}}{a^{3}}+\frac{\Omega_{r, 0}}{a^{4}}+\Omega_{\Lambda, 0}+\frac{\Omega_{K, 0}}{a^{2}}\right)
$$

where the "0" suffix implies current time.

Where $\Omega \equiv \frac{\rho}{\rho_{\text {crit }}}=\left(\frac{8 \pi G}{3 H^{2}}\right) \rho$

In (13) again we have matter, radiation, cosmological constant, and curvature terms.

From (12), we have

$$
\Omega_{\text {tot }} \equiv \Omega_{m}+\Omega_{r}+\Omega_{\Lambda}=1-\Omega_{k}
$$

Carrol et al. (1992) [73] have carried out a comprehensive review of the efforts to calculate or assess the vacuum energy $\Omega_{\Lambda}$ and Carrol (2001) [74] discuss various model universes.

According to Carrol (2001), the relative contribution of each of these components changes with time as follows

$$
\Omega_{\Lambda} \propto a^{2} \Omega_{k} \propto a^{3} \Omega_{m}
$$

At the current time, based on observations we have

$$
\begin{gathered}
\Omega_{\Lambda 0}=0.7, \\
\Omega_{m 0}=0.3, \\
\Omega_{r 0}=5 \times 10^{-5} .
\end{gathered}
$$

Going by relationship (15) $\Omega_{m}$ and $\Omega_{\Lambda}$ should diverge rapidly from their initial conditions with the evolution of the universe but out of a strange coincidence in present times both are of the similar order of magnitude, which is also puzzling and another major problem of SMC.

\section{Review of Efforts at 5DU}

The concept of a five dimensional universe (5DU) or a $(4+n)$ DU as against a four dimensional universe (4DU) has been around for almost 100 years. Along with the 4DU SMC, an independent 5D treatment of the cosmos also evolved but is not as widely accepted as the SMC. The 5DU attempts are related to the quest for unified field theory and the efforts can be traced to Weyl (1918) [75], who in the theory he developed, tries to unify gravitation and electromagnetism. Weyl makes a profound and a bold statement: 


\section{"all physical quantities have a world-geometrical meaning"}

According to Kaluza (1921) [20], Weyl based on the concept of infinitesimal parallel-transfer in Riemannian geometry, arrives at a kind of a fundamental metric vector, interpreting it as the electromagnetic four-potential $q \mu$. Kaluza in an attempt to find a common ground for electromagnetic and gravitational fields, it seems for the first time, introduces the concept of a real $5^{\text {th }}$ dimension. Klein (1926) [76] made significant additional contributions to the 5DU concept and the theory is now known as Kaluza-Klein theory.

Kaluza-Klein came up with the equations of motion of an electrified particle as equations of geodetics belonging to line element as given below:

$$
\mathrm{d} \sigma=\sqrt{\left(\mathrm{d} x^{0}+\beta \phi_{i} \mathrm{~d} x^{i}\right)^{2}+g_{i k} \mathrm{~d} x^{i} \mathrm{~d} x^{k}}
$$

where $x^{1}, x^{2}, x^{3}, x^{4}$ are the co-ordinates of ordinary space-time with the line element $g_{i k} \mathrm{~d} x^{i} \mathrm{~d} x^{k}$, while $x^{0}$ is a fifth co-ordinate, and the $\phi_{i}$, are the four co-variant components of the electromagnetic potential vector. Equation (1) clubs the $5^{\text {th }}$ coordinate with the electromagnetic potential vector. According to Klien, five dimensional space is assumed to be closed in the direction of the $5^{\text {th }}$ dimension $x^{0}$ with a certain period $l$, for which a small value of the order of $10^{-30} \mathrm{~cm}$ is obtained. The periodicity and the smallness of the value in $5^{\text {th }}$ dimension results in it being completely missed out in experiments. The size of $10^{-30} \mathrm{~cm}$, almost of the order of Planck length will be difficult to detect, and thus $5^{\text {th }}$ dimension, if it exists, remains undetected.

According to Wesson (2012) [21], the Einstein's Equivalence Principle (EEP) could be a direct consequence of the existence of an extra dimension and that energy density and pressure (matter) is a property of $4 \mathrm{D}$ spacetime, owing its existence to the fifth dimension. Wesson and others (Lidsey et al.) [77] refer to Campbell's embedding theorem, which implies that all solutions to the $\mathrm{n}$-dimensional Einstein field equations with arbitrary energy-momentum tensor can be embedded, at least locally, in a spacetime that is itself a solution to $(\mathrm{n}+$ 1)-dimensional vacuum GTR. Wesson [78] comments that the most telling objection to $5 \mathrm{D}$ universe is that after many years of investigation, there is still no empirical proof of the existence of even one extra dimension.

Wesson and Leon [79] present derivation of a general equation of motion of a particle in space-time augmented with one additional dimension. They conclude that Kaluza-Klien theory is a logical extension of Einstein's theory and that whether the universe is $4 \mathrm{D}$ or $5 \mathrm{D}$ was subject to astrophysical deductions.

According to Wesson (2008) [80] Einstein's equations with matter in $4 \mathrm{D}$ are a subset of the Ricci-flat equations for apparently empty space in 5D. Wesson considers $5 \mathrm{D}$ space with $5 \mathrm{D}$ line element

$$
\mathrm{d} S^{2}=g_{A B} \mathrm{~d} x^{A} \mathrm{~d} x^{B}
$$

where $A, B=0,1,2,3,4$

$$
g_{00}=\varepsilon \Phi^{2}
$$

where $\varepsilon= \pm 1$ allows for both spacelike and timelike dimensions, and 
$\Phi=\Phi\left(x^{\alpha}, \ell\right)$ is the Scalar potential. The 5D interval is given by

$$
\mathrm{d} S^{2}=g_{\alpha \beta}\left(x^{\gamma}, \ell\right) \mathrm{d} x^{\alpha} \mathrm{d} x^{\beta}+\varepsilon \Phi^{2}\left(x^{\gamma}, \ell\right)
$$

According to Wesson, the rest mass is analogue of the proper distance in the fifth dimension and can be given by

$$
m \equiv \int \Phi\left(x^{\gamma}, \ell\right) \mathrm{d} \ell
$$

Equation (19) is a fundamental concept that presents some form of similarity between mass and distance, which we also discuss in the later section.

It is also suggested [81] that the cosmological constant $\Lambda$ scales with mass, $m$ as follows:

$$
\begin{gathered}
\Lambda \sim m^{2} \text { : for small } m \\
\Lambda \sim 1 / m^{2} \text { : for large } m
\end{gathered}
$$

Randall and Sundrum (RS) (1999) [19] and Arkani (1998) [82] have also considered a higher $4+n$ dimensional spacetime to address the hierarchy problem [83]. The hierarchy problem is related to a very small ratio between electroweak scale $m_{E W} \sim \mathrm{TeV}$ and the Planck scale $M_{P L} \sim 10^{15} \mathrm{TeV}$. The ratio $m_{E W} / M_{P L} \sim$ $10^{-17}$. RS suggest that if spacetime is considered as $4+n$, then the effective $4 \mathrm{D}$ planck scale is determined by $(4+n) \mathrm{D}$ Planck scale. According to them, the source of the hierarchy is an exponential function of the compactification radius. They point to small exponential factor as the source of the large hierarchy between the observed Planck and weak scales and the four-dimensional masses on the visible brane depend on the background metric.

Arkani (1998) [82] propose that $m_{E W}$ be considered as the only short distance scale in nature and accordingly they conclude that the $4 \mathrm{D}$ universe should have a "thickness" $\sim m_{E W}^{-1}$ in the extra " $n$ " dimensions.

To summarize, the $(4+n)$ DU proponents, seem to hypothesize $4 \mathrm{DU}$ as a very thin P-brane within a $(4+n)$ DU. Some of the shortcomings of SMC seem to get resolved and there is a new notion for mass, which is related to the geometry in $(4+n)$ DU. However, the fact that there is no observed evidence or experimental confirmation of $4+$ dimensions is holding back acceptance of a $4+$ dimensional model.

In the next sections we undertake treatment of some of the aspects of time and other $4+$ dimensions, which bring in an element of additionality to SMC conceptual framework.

\section{Extended Theory of Gravity (ETG)}

A viewpoint on the late-time acceleration of the universe and the aspect of dark matter is that at large scales, the Einstein's gravity model in GTR breaks down, and a more general action describes the gravitational field. According to Salvatore and Mariafelicia (2011) [22], Einstein equations are no longer a good test for gravitation, unless the matter side of field equations contains some kind of exotic matter-energy which is the "dark matter" and "dark energy" side of the 
universe. According to Corda (2009) [24], quantization of gravity has been a challenging proposition, which prevents the unification of gravity with other known forces. Corda [24] is of the opinion that gravity is different at various scales and a room for alternative theories is present. He proposes advanced projects on detection of gravitational waves (GW) to detect "+", "X", and "Scalar" polarizations using advanced and more sensitive interferometers. If only only "+" polarizations (which are additive) and "X" polarizations (which cancel out) are discovered, the GTR can be considered as definitive. However, if a third polarization is detected by GW interferometers, General Relativity can be ruled out as definitive theory of gravity.

\section{Time}

Understanding of time is an important aspect of cosmology, philosophy and physics in general and a review of the "time" concepts in the context of SMC is called for. However, on the other hand, though immensely important in the study of dynamic systems and the past and future states of the universe, an involved discussion on time e.g., like Penrose [1], may be beyond the main theme and topical bounds of this paper. Here we briefly review some of the relevant aspects of time.

In SMC and in fact in modern physics, "time" is considered as a dimension but in order to treat "time" in the same manner as the other space-like dimensions, we face quite a bit of philosophical and conceptual difficulty. We are not able to visualize it as a dimension in the same manner as the other space coordinates and as a result, physicists usually make distinction between "timelike" and "spacelike" dimensions.

At the most fundamental level, the "passing of time" is something we sense in not only observing but also in being a part of the natural processes. All processes take time or give us a feeling of the passage of time - be it the heating of water till its boiling point, passing of days, nights and seasons, aging, etc. Though time is not sensed in the same manner as space dimensions, it can be considered a dimension because any event can be assigned a unique space $\left(x^{1}, x^{2}, x^{3}\right)$ coordinates and an instant of time $\left(x^{0}\right)$. In this manner it is possible to define four dimensional interval as:

$$
s^{2}=\eta_{v \mu} \Delta x^{\mu} \Delta x^{v}
$$

where $\eta_{00}=-1$ (assuming $c=1$ ), also known as the Minkowski space.

Albert Einstein addressed some but not all the crucial aspects of this enigmatic dimension. He addresses "simultaneity", which is also one of the cornerstones of GTR.

\subsection{Arrow of Time}

The "direction" (if we can call it "direction") of the passage of time is always sensed by us as flowing from past to future, also known as the "arrow of time." However, in GTR and Newtonian laws and equations, time is reversible and 
there is no arrow implied in these laws. Stephen Hawking [84] introduces three kinds of the arrows of time, i.e., 1) the thermodynamic, 2) the psychological, and 3) the cosmological. Roger Penrose [1] considers additional conditions w.r.t. the arrow of time - 1) decay of $\mathrm{K}^{\circ}$-meson, 2) quantum-mechanical observations, 3) entropy increase, 4) retardation of radiation, 5) psychological time 6) expansion of the universe 7) black hole v/s white hole.

\subsubsection{Thermodynamic Arrow}

The thermodynamic arrow is related to the second law of thermodynamics, according to which entropy always increases with time or in other words everything moves from an ordered state to a disordered state. With respect to the initial state of the universe as seen in $\mathrm{CMB}$, Penrose finds it paradoxical that in the initial state we have high randomness (read high entropy), and inflation onwards the arrow of time points from low entropy to high entropy, this has led him to the CCC concept. The cosmological arrow of time, which is the expansion of the universe, is also a manifestation of the thermodynamic arrow.

\subsubsection{Psychological Arrow}

The psychological arrow is what we would observe and sense. From the relativistic point of view as well as the Schrodinger's equation, the observer is a very important aspect of modern physics. Hence as long as this sensation of the passage of time is not a creation of our imagination, it has a real meaning in physics. One should keep in mind that what we sense as time may or may not be just one more dimension time but could be more than one dimensions, an aspect we take up in a later section of the paper.

\subsubsection{Time Reversibility and Information Loss}

Time reversibility and associated issues with information is an interesting aspect we touch upon through the example below:

Let us assume, the universe expands and then undergoes a big crunch process so that there is also a time reversal and things flow back from high entropy to low entropy. Though the likelihood of any such thing happening is debatable [85], it has been researched and discussed [86] [87]. Therefore, For the sake of the discussion, we assume that one doesn't get into any of the complexities associated with such time reversal and every event is traced back exactly as it happened. If this does not happen, then it will be difficult to justify the condition of "high entropy to low entropy" (required in time reversal). We are looking at water reversing into clouds, spilled milk coming back into the milk pot etc. An observer, Mr. X witnessing this process of the universe winding backwards has a difficulty in handling information as he would be progressively losing memory with time reversal. In fact not only in the observers mind but also in all natural systems in the universe memory will be getting regressively erased. Let's assume Mr. X who kept a kettle of water to boil at a certain time and a certain day in 2021 in the universe characterized with low entropy to high entropy flow. Say, several billion years later when the time reversal is taking place and Mr. X ap- 
proaches the same kettle at the same time (reversed) and same place, his memory of a future he had already lived would have been erased and with every reversing moment, his memory of a future in which he existed will be getting erased but he has the memory of the distant past, where he is now headed. There is no way for Mr. $\mathrm{X}$ to know that he is in a time reversing universe. He will continue to slide from high entropy to low entropy state. All the time as long as he has memory of a previous epoch, he will imagine the universe is expanding but actually, he would be facing the big crunch. In reality, he will go back to school, be a child again and vanish into the womb. A time reversing universe can also be a possibility for us. Though a philosophy-like question, it is a real question of physics.

Somewhere, in this discussion we need to factor-in the loss of information with time reversibility. In the "low entropy to high entropy" universe, the one we assume we are living in, information is created and stored. However, in a "high entropy to low entropy" time reversing universe existing information will be lost and all that is stored or recorded will be erased.

\subsection{Relativistic Aspect of Time}

There is also the relativistic aspect to time. The constancy of the velocity of light in vacuum, based on Maxwell's electro-magnetic field equations (Equation (24)) and assumed as universally true by Einstein has led us to Lorentz transformations, according to which at relativistic velocities, time dilation and length contraction happens as given by Equation (21):

$$
\left(\Delta x^{\mu}\right)^{\prime}=\Lambda_{v}^{\mu} \Delta x^{v}
$$

where $\Lambda_{v}^{\mu}$ is given by

$$
\left(\begin{array}{cccc}
\gamma & -\gamma v_{x} / c & 0 & 0 \\
-\gamma v_{x} / c & \gamma & 0 & 0 \\
0 & 0 & 1 & 0 \\
0 & 0 & 0 & 1
\end{array}\right)
$$

and $\gamma$ is given by

$$
\gamma=\frac{1}{\sqrt{1-\frac{v^{2}}{c^{2}}}}
$$

while the Maxwell's relation is given by:

$$
C=\frac{1}{\sqrt{\mu \varepsilon}}
$$

where $\varepsilon$ is the permittivity, and $\mu$ is the permeability in vacuum.

On the other hand in GTR, time elongation in the presence of gravity is given by [88]:

$$
\Delta t^{\prime}=\Delta t_{f} \sqrt{1-\frac{G M}{r c^{2}}}
$$


In case of a very strong gravity such as in a black hole singularity or the big bang singularity, theoretically, the time can stop elapsing.

As far as relativistic concepts are concerned, we notice two things 1) any motion (which is always relative between respective frames of reference) transforms space-time coordinate system to cause time dilation and shrinkage of space along the direction of relative motion, and 2) presence of mass (rather energy) deforms spacetime by means of gravity. Figure 2(a) and Figure 2(b) show how a time interval of $1 \mathrm{~s}$ in free space (region of space free of gravity) transforms to ellapse slowly due to relative velocity and mass respectively. Similarly length contraction due to motion and gravity respectively is shown in Figure 2(c) and Figure 2(d).

It is interesting to note that the trajectory of both time and space dilation and contraction under Lorentz transformation on account of relative motion and under Einstein's equations on account of gravity are similar. Moreover, if we plot "inverse of time" $(1 / t)$, the behavior of inverse time is similar to that of physical dimensions (length). This leads us to a suspicion that the inverse of time is a space like dimension, which is a real dimension and our observational and sensory system delivers to us an inverse of the dimension. One cannot miss noticing an uncanny similarity between how "mass" and "relative velocity" transform time and length as seen in Figure 2. Since, both "mass" and "velocity" are manifestations of energy, this may be due to a deeper underlying reason than a mere mathematical coincidence.

We have already quoted Wesson [80] [81] above where he relates mass to length in $5^{\text {th }}$ dimension. Figure 2 could be an indicator in support of the $5^{\text {th }}$ dimension.

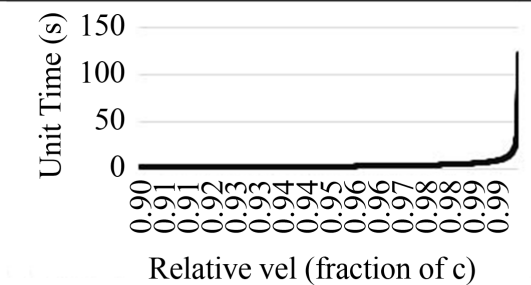

(a)

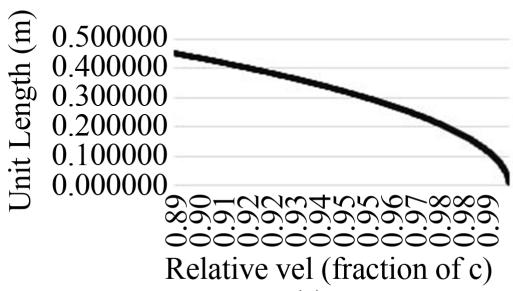

(c)

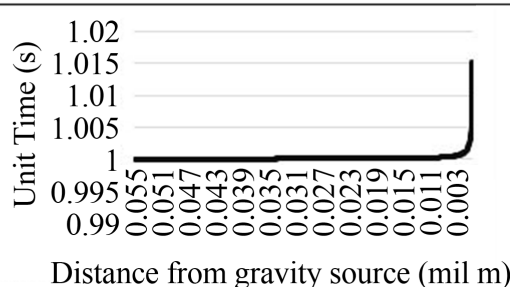

(b)

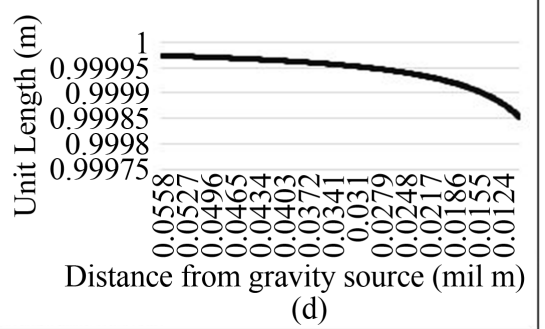

Figure 2. Lorentz and gravity time alongation and length contraction. (a) The relative velocity increases till close to $\mathrm{C}$ and time interval of $1 \mathrm{~s}$ takes longer ellapse (time elongation), Motion; (b) Gravity increases as a body approaches a large gravity source time elongation takes place, Mass-gravity; (c) The relative velocity increases and length contracts, Motion, Based on Lorentz Transformations; (d) Length contraction under gravity, Motion, Based on GRT. 
At sub-atomic levels, because of the relativistic considerations, we obtain a different perspective of time. To a sub-atomic observer, who is at rest w.r.t. the nucleus, at some positions, the electron's time should be more dilated than at some other positions. If " $V$ " is the tangential velocity, the component of " $V$ " at its maxima will be $V \cos (0)$ and at its minima will be $V \cos (\pi / 2)$.

The factor $\gamma$ used in length contraction and time dilation at any position w.r.t the sub atomic observer will be given by:

$$
\gamma=\frac{1}{\sqrt{1-\frac{(v \cos \theta)^{2}}{c^{2}}}}
$$

and time dilation by

$$
t^{\prime}=t *\left(\frac{1}{\sqrt{1-\frac{(v \cos \theta)^{2}}{c^{2}}}}\right)
$$

where $\theta$ is the angle as shown in Figure 3.

The variance in radius as seen by the subatomic observer can be calculated for the hydrogen atom to be of the order of $10^{-15} \mathrm{~m}$ and that in the 'time period' of the order of $10^{-22} \mathrm{~s}$. This is much larger than Planck length and time but much smaller than wavelength of visible light or even X-rays.

In reality there will also be the spin of the electron, the electromagnetic field, and the mass-all of which will fluctuate with time dilation, adding to the complexity. Due to the periodic fluctuations, for the sub-atomic observer at rest, the behavior of electron will be a wave form, which can be expressed in the form of a standing wave function. It is a remarkable conclusion as here we had not assumed the well established wave nature of electron but the relativistic considerations lead us to a wave form, even if we assume an electron as a classical Newtonian particle and not a quantized wave-particle.

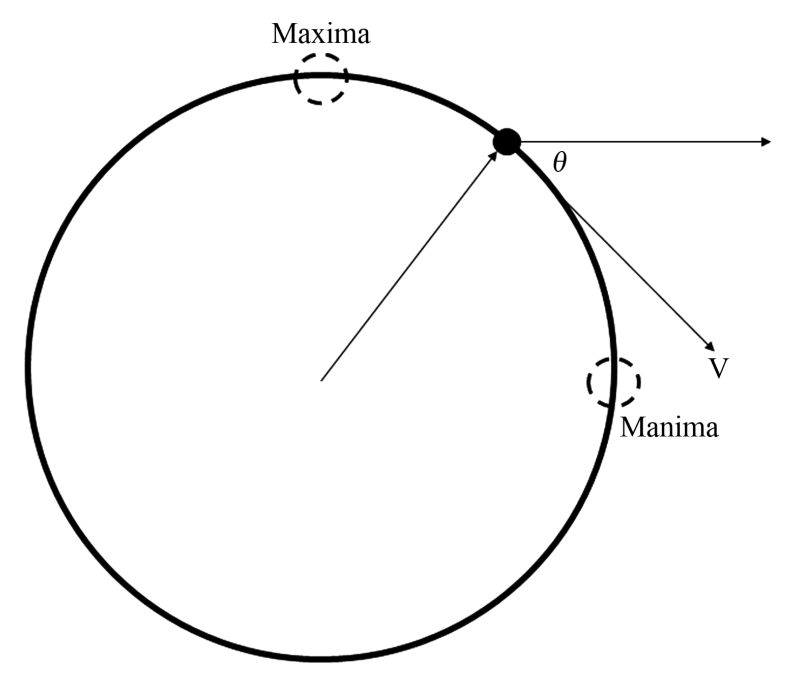

Figure 3. Electron as a kinematic particle. 
Now let's examine this from the viewpoint of a macroscopic observer. For example, let's take the example of any solid object, say a brick. Inside the brick, there are innumerable sub-atomic particles, which w.r.t. to the brick at rest, are in time frames in accordance with their sub-molecular and sub-atomic movements and rotations.

Now, if the brick is observed at an instant of proper time " $\vec{~}$, can we say that the position of the brick on the time dimension is entirely defined by " $t$ "? The sub-atomic particles seem to exist in a band of " $t \pm \delta t$ ", where, " $\delta t$ " relates to the sub-atomic time oscillations that happen even within an atom, as discussed above. In other words, the brick exists in three dimensions (length, breadth and height) and a time interval, $\delta$ t. From this we can conclude that the brick has a spread in the fourth dimension. The same analogy can be applied to large cosmic bodies that are spinning and are also revolving in an orbit. Here, if the velocities are non-relativistic, any such implication is miniscule from a velocity perspective. However, the relativistic gravitational aspect will render four dimensionality to these bodies on a macro scale. For example, Sun may be spread out over a time interval of $10 \mathrm{~s}$.

It may be noted that in the relativistic treatments also, all bodies and objects are treated as three dimensional but the spacetime is considered as four dimensional. This aspect brings about a fundamental change in the way we look at the universe. In GRT, we have a 4DH (space-time) but all observed objects are 3 dimensional. The $4 \mathrm{DH}$, though it is curved presumably in space-time, has no thickness in the $4^{\text {th }}$ dimension. Though, in reality, there is a certain "time-interval-thickness", which may vary in accordance with the body or the object. In case of a brick, it may be of the order of $\sim 10^{-10} \mathrm{~s}$ but in case of sun, it may be 10 $s$, and in case of a black hole at the center of the milkyway, going by the singularity consideration it should be infinite or in any case a very large value of the order of hundreds of thousands of seconds. Such time interval thickness of any object from a GRT perspective can be calculated considering its mass and radius.

The above example also shows that for a macroscopic observer, even if we assume he has very powerful tools to observe subatomic phenomena, he cannot exactly measure the time, position, mass, momentum and the electromagnetic fields. Due to the oscillatory aspect associated with time dilation in this case, the only picture of an electron that one can arrive at is that of a standing wave. Thus time dilation in the subatomic world links us to two important theories, both well established with ample evidence, and were postulated almost 100 years ago-one is the De Broglie's wave particle theory and the other Heisenberg's uncertainty principle. This, however, has to be justified with greater rigor and evidence collection before one can claim to have somewhat demystified these established theories.

In the context of modern cosmology, our formulations are like those in the bulk and brane theory [1] [89], according to which particles corresponding to electromagnetic, weak and strong interactions are confined on some hypersur- 
face with sub-atomic thickness and a bulk, a macroscopic dimension. The time "interval thickness" is in the nature of brane world scenario, as in Randall-Sundrum (RS) and other $(4+\mathrm{n}) \mathrm{D}$ models. This seems to be the second evidence of a $5 \mathrm{DU}$.

The other important aspect in the above example is that of the difficulty for the macroscopic observer in determining the exact mass, velocity, momentum and position of the electron because the observations are based on macroscopic course-grain time. Therefore, the observer will have to do with the probabilities and wave function with a Schrodinger's equation of the standing wave, typically given in its time independent form by:

$$
\frac{\hbar^{2}}{2 m} \frac{\mathrm{d}^{2} \psi(x)}{\mathrm{d} x^{2}}+(E-V(x)) \psi(x)=0
$$

where $\psi(x)$ is the wave function $V(x)$ is the potential energy term.

It is natural to assume that there should be a scale, over which macroscopic order takes over or in other words below which, we have the sub molecular and sub-atomic microscopic realm. This scale should be related to the least coarse grain space-time that we can observe.

One can hypothesize that certain phenomenon can only be observed, if their occurrance is above a certain coarse grain threshold. This may have an implication on the quantum concepts.

This example above, leads us to following important conclusions:

1) The proper time observed with regard to a body at rest or in motion w.r.t. the observer, is a coarse grain time as compared to the time position of the sub-constituent (sub-atomic) particles of that body.

2) This further implies that anybody has a certain "time interval-thickness" associated with it or in other words, the body exists in a certain slice or segment of time and not just an instant. This should hold true irrespective of the frame of reference.

3) Thus we can say with reasonable confidence that any object in the universe is at least four dimensional.

There is nothing new about point 3 ) above since according to string theory, there are upto 26 dimensions. However, in the working of classical, relativistic, and (in some measure) quantum theories-bodies or objects, particularly if they are non-relativistic and macroscopic are treated as three dimensional though space-time is considered as four dimensional. Our discussion above, should have implications both, on cosmological and quantum scales.

In the discussion above, we have treated time as a dimension. It is, however, possible that our sense of the passage of time is a combined effect of many dimensions other than the three space dimensions.

\subsection{Energy Flow and Causality}

Another aspect associated with the "arrow of time" is the flow of energy and causality. Causality is related to the flow of energy in some form or the other be- 
cause nothing can actually be caused in the universe without a play of energy. This reasoning appears quite logical. Now this flow of energy, at least in the macroscopic world is governed by gradients and always flows from high potential to low potential.

This in fact should be a universal law and is related to the "arrow of time" from low entropy to high entropy. One can always try to push energy in a reverse direction such as pumping of water to an elevated place but since there cannot be any perfect and 100\% efficient machine, we always have loss and dissipation, which adds to the disorder. Thus in everything that happens in the universe, we have energy flow from high potential to low potential and an increase in entropy.

\section{Dimensionality of the Universe}

On a cosmological scale, universe is assumed to be 4 dimensional but on the plank scale $\left(10^{-35} \mathrm{~m}\right) 10$ or even 26 dimensions have been proposed. Our existence, to the best of our understanding and life experiences is physically limited to three dimensions. However, two observations awaken us to the possibility of higher dimensions, one of them is the constancy of the velocity of light and the other one the red shift. Constancy of velocity of light led scientists to the concept of space-time continuum (Einstein, Lorentz, Minkowski). On the other hand, the red shift [90] is the only direct and the most significant observation that implies stretching of the very fabric of the space-time continuum. The mutual receding of the galaxies is best explained by invoking a $4^{\text {th }}$ dimension.

With regard to expansion of the universe and its dimensionality we came to some interesting conclusions strengthening the case for the fifth dimension. Here we present a general case of dimensionality and expansion in the form of the "Theorem of the Dimensionality of the Expanding Universe."

According to GTR, time and space are intertwined or together as space-time, which means space and time do not exist independently as was assumed in Newtonian physics. If we were to consider space-time relationship in curved space along a geodesic, the time gets elongated as one approaches the source of gravity. This, however, actually means that the time interval shrinks. If we take the example of our solar system, an interval of two seconds at heliopause might become 1 second close to the surface of the sun (assumed and not calculated). We call it time elongation because close to the surface of the sun, the passage of time is slower. Likewise from heliopause to the surface of sun, any standard measure of length will also shrink (Section 5.2). In fact, in the case of black holes, inside the Schwarzschild radius [91], space and time, if they were viewed as separate entities, get swapped. Thus, we go with the well established fact that the spacetime is the fabric of the universe, and, therefore, what is stretching or expanding is this fabric. If we were to compare the expansion of the universe with the analogy of an expanding balloon, then the membrane (skin) of the balloon is the universe and the space-time fabric and each point on this balloon is a stellar object or a galaxy. 
Since the expansion of the universe is the key observation leading us to higher dimensionality, and though it is a very simple concept, we feel it is important to define it with clarity in the context of cosmology. We define expansion in general as follows:

Expansion of an $N$ dimensional hypersurface $(N D H)$ of an $M$ dimensional universe $(M D U)$ is defined as a process involving distancing of every point on $N D H$ from every other point on it at a certain rate w.r.t. an independent dimension, which is not contained in $\mathrm{NDH}$

In order to gain deeper insights with regard to the dimensionality of the universe, we begin not with a 4 dimensional universe but with the very fundamental levels of 1 and 2 dimensions. Say, we have a standalone straight line "L", which contains a segment $A B$ as shown in Figure 4 below. This is all we have. There is no other dimension. $\mathrm{AB}$ is then our universe, a one dimensional hypersurface and universe (1DH and $1 \mathrm{DU})$ that has only one axis, $x^{1}$. A has the coordinates $x_{1}^{1}$ and $\mathrm{B}$ has the coordinate $x_{2}^{1}$.

Now we pose a question-can AB expand? The answer is "no" because AB has nowhere to expand. " $X$ " as $1 \mathrm{DU}$ is a static frozen universe in perpetuity (if we can use the term perpetuity in the absence of time) and there is no other dimension in a $1 \mathrm{DU}$ for $\mathrm{AB}$ to expand upon or with respect to. However, if we now enhance 1DU with an additional dimension $x^{2}$ so that we now have a plane in which " $\mathrm{L}$ " is a line segment. We can now consider expansion of AB to A'B'. If we assume the origin to lie on the original $x^{1}$ axes, the new coordinates of $A^{\prime}$ and $\mathrm{B}^{\prime}$ in the $2 \mathrm{DU}$ are $\left(x_{3}^{1}, x_{3}^{2}\right)$ and $\left(x_{4}^{1}, x_{4}^{2}\right)$ respectively. Now even though we have not necessarily provided, what we call a "time like dimension", expansion of $A B$ can be imagined in the plane $x^{1}-x^{2}$ to $A^{\prime} B^{\prime}$.

The rate at which $A B$ expands will be given by $d A B / d x^{2}$, where $d A B$ is the incremental increase in length of $\mathrm{AB}$ (expansion) and $\mathrm{d} x^{2}$ the corresponding increment in the $x^{2}$ dimension. The second dimension, in a sense acts like "time" and could in fact be "time" as well. As AB expands from state $\left\{\left(x_{1}^{1}\right)-\left(x_{2}^{1}\right)\right\}$ to

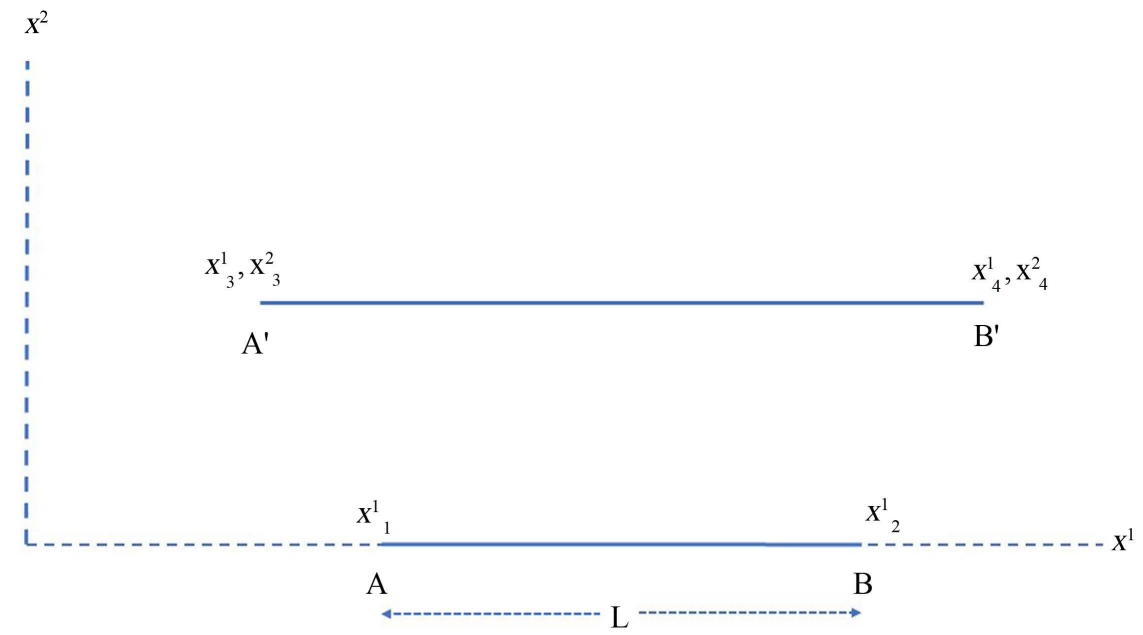

Figure 4. Expansion of 1 dimensional line in 2 dimensions. 
$A^{\prime} B^{\prime}\left\{\left(x_{3}^{1}, x_{3}^{2}\right)-\left(x_{4}^{1}, x_{4}^{2}\right)\right\}$, every point between A and B gets distanced from every other point in accordance with the definition of expansion provided above.

Likewise, if we apply the same analogy to the expansion of a 2D hyper-sphere (circle) as shown in Figure 5, we find that it cannot expand just in two dimensions and needs another dimension to expand into. In a $2 \mathrm{D}$ hyper-sphere, the expansion can happen in two ways. One is an expansion of the circle along the

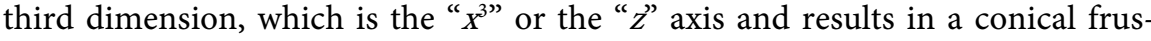
tum. The other way of expansion will be in time. if a circle is expanding in time we would normally consider rate of change of the radius of a hypersurface w.r.t. time i.e., $\mathrm{d} r / \mathrm{d} t$.

In the next case, we assume $A B$ to be a curved segment. Now since $A B$ is curved, it has a two dimensional form and exists as $2 \mathrm{DH}$ but now for $\mathrm{AB}$ to expand, we need a third dimension, which provides $\mathrm{AB}$ with the additional dimension to expand upon-either time $\left(x^{0}\right)$ or $x^{3}$. In this manner we can graduate to other forms and dimensions. A circle in $2 \mathrm{DH}$ can only expand in 3DU, a balloon or a $3 \mathrm{D}$ sphere in $3 \mathrm{DH}$ can only expand in $4 \mathrm{DU}$.

Now in the case of the universe, it is in the form of a $4 \mathrm{DH}$ space-time hypersphere. As concluded in section 5.0, every object in the universe is 4 dimensional and as discussed above, it is established that spacetime continuum is the fabric of the universe. Therefore, by the logic mentioned above, this $4 \mathrm{DH}$ universe can only expand in a 5DU.

In general the difference between $\mathrm{NDH}$ and an $\mathrm{MDU}(\mathrm{M}>\mathrm{N})$ should be recognized. $\mathrm{NDH}$ is what contains the contents of the observable universe, which is matter, energy, radiation, gravity, spacetime, etc. In case of 1DU example above, it just contains $\mathrm{AB}$. MDU by virtue of $\mathrm{M}>\mathrm{N}$ provides the additional dimension for the NDH to expand upon but may or may not contain anything in addition to NDH. One must keep in mind the general case that allows expansion as well as shrinkage(negative expansion). In short:

$$
\mathrm{NDH} \in \mathrm{MDU}
$$

Before proceeding further, we record this fact as a theorem, the Theorem of the Dimensionality of the Expanding Universe as follows:

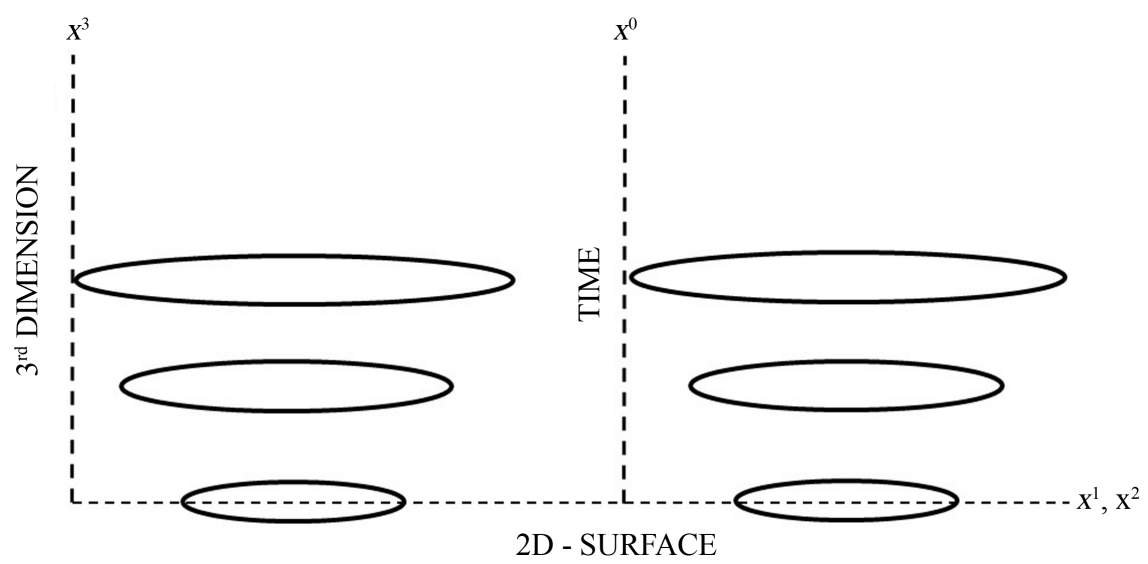

Figure 5. Expansion of a circle from 2D plane to 3D space or 3D Space-time. 
Given any $N$ dimensional Universe Hypersurface $(N D H)$, for it to undergo expansion, there must exist an $(N+1)^{\text {th }}$ dimension and at least a $(N+1) D U$.

The proof is straight forward since in expansion, as a process, every point in $\mathrm{NDH}$ must distance itself from every other point in accordance with a certain rate change with respect to a certain dimension $x^{k}$. The $x^{k}$ dimension cannot be a part of the $N$ dimensions but an additional $N+1^{\text {th }}$ dimension.

It is well established in GRT and that the universe is a $4 \mathrm{D}$ space-time curvature (hyper-sphere), which has been expanding since the epoch of big bang, therefore, from the theorem of the Dimensionality of Expanding Universe, we conclude that there must exist a $5^{\text {th }}$ dimension. Moreover, since the expansion is happening on a cosmological scale, the $5^{\text {th }}$ dimension should also be on a cosmological scale. Hence, we are looking at a non-compact $5^{\text {th }}$ dimension or the bulk.

The implication is significant from a cosmological perspective that the universe, generally accepted and perceived as 4 dimensional, has a $5^{\text {th }}$ dimension to it. A 5DU has implications on big bang, inflation and SMC.

This leads us to another question, which should be resolved - Is the time interval thickness, we discussed at sub-atomic level, the same time dimension as the one on the cosmological scale? We think there is room for such a thought because we have agreed that by the theorem of dimensionality, the universe had 5 dimensions and we also pointed out that the feeling of the passage of time that we experience could be a combination of many dimensions. Though it appears that we are dealing with the same time dimension at the microscopic and cosmological levels, at this juncture, we do not attempt to answer this question.

\section{Expanding 4D-Brane}

In a previous section (5.0), we established that every physical object is 4-dimensional, with the fourth dimension being a slice or a membrane of the order of subatomic dimensions. In section 6.0, we establish that the universe is 5DU. With this understanding, if we go back to big bang, we can reimagine it in a somewhat different manner from that in the case of a $4 \mathrm{DU}$. Going by the theorem of dimensionality of the expanding universe, the big bang was an event leading to a 4DH hypersphere that is even today in a state of accelerated expansion in a $5 \mathrm{DU}$ and the universe we observe, exists on a $4 \mathrm{DH}$ layer or a mem(brane) of this 5DU (we shall refer to it as "our brane"). The 5D and brane concepts are established and have been pursued by a number of researchers over the last 100 years (Randall and Sundaram (1999) [19], Kaluza (1921) [20], Wesson (2012) [21]). Our work presented here, further strengthens the case for the $5^{\text {th }}$ dimension. It is possible to consider the $5 \mathrm{DU}$ as one of the alternatives to cosmological models with at least the same level of confidence as CCC and Guth's false vacuum concepts.

If indeed it is agreed that $5 \mathrm{DU}$ is as viable an alternative as CCC and inflation, we find that it might also offer solutions to some of the lacunae of SMC. In fact, 
we may have to set aside some of the ideas that have been pursued so far with regard to inflation, $\Lambda$, dark energy and dark matter.

We have no knowledge of what happened in the aftermath (we refer to the time after the big bang as the "aftermath") of the big bang, as our brane expanded. Apart from our brane, were there other branes, before or after us? Is the analogy similar to that of an expanding balloon with a single thin membrane or that of many layers, something that should be seen as nested hyper-involutes of a $4 \mathrm{D}$ Sphere. Involute because after the big-bang, the $4 \mathrm{D}$ branes expand and as a result, a photon will trace out an involute like trajectory.

Was there more energy ejected in the big-bang after "our brane" passed through that epoch? If the rate of the expansion of our brane's radius is more than the speed of light and if the aftermath radiation is travelling at the same effective rate as the speed of light, then we shall never know as to what happened unless somehow, the "aftermath" matter and energy are detected.

There are quite a few possibilities that are listed in Table 3 below. We have assumed " $R$ " as the rate of expansion of the brane.

The other reason for us to not detect any light coming from other branes could be due to the fact that the light itself as a wave or perturbation in electromagnetic field is a phenomenon of the brane and remains restricted to it. This has to do with the fact that light travels along a geodesic in the $4 \mathrm{DH}$ and that geodesic is defined in each brane. The light that we see from far off galaxies $\sim 10$ billion light-years have traced out a path along an hyper involute as our brane expanded and there is a possibility of many similar branes and layers of such hyper involutes.

If we take the multiple expanding $4 \mathrm{D}$ branes as the model, the entire structure turns out to be different. With other branes, in the pre-inflation stage, the

Table 3. The brane possibilities in 5DU aftermath.

\begin{tabular}{|c|c|c|c|}
\hline Possibility & Sub-Possibility & Implications & $\begin{array}{c}\text { Universe } \\
\text { Parameters }\end{array}$ \\
\hline Only one brane & nill & $\begin{array}{c}\text { Why accelerated expansion? } \\
\text { What is Source of dark } \\
\text { matter and energy? }\end{array}$ & $\begin{array}{c}\text { As } \\
\text { assessed now }\end{array}$ \\
\hline \multirow{4}{*}{$\begin{array}{l}\text { Other branes } \\
\text { in the aftermath }\end{array}$} & A) $\mathrm{R}>\mathrm{C}$ & $\begin{array}{l}\text { We may never observe other } \\
\text { branes in the aftermath }\end{array}$ & Higher Mass \\
\hline & B) R $<$ C & $\begin{array}{l}\text { We should have received } \\
\text { radiation from the Aftermath } \\
\text { or will receive in future }\end{array}$ & Higher Mass \\
\hline & C) $\mathrm{R}=\mathrm{C}$ & -do- & Higher Mass \\
\hline & $\begin{array}{l}\text { D) Radiation in } \\
\text { aftermath travels } \\
\text { at different speed } \\
\text { than } C\end{array}$ & Again $-\mathrm{A}, \mathrm{B}, \mathrm{C}$ & Higher Mass \\
\hline $\begin{array}{l}\text { Other branes } \\
\text { preceding ours }\end{array}$ & \multicolumn{2}{|c|}{$\begin{array}{l}\text { Possibilities A,B, C, D w.r.t Preceding } \\
\text { brane with our brane in the aftermath }\end{array}$} & Higher Mass \\
\hline
\end{tabular}


matter-energy density shall be far in excess of whatever we have computed. The inner layers should be exerting outward pressure on our brane and quite possibly our brane exerts a pressure on a brane beyond ours. This may give a new meaning to $\Lambda$.

In the $4 \mathrm{D}$ SMC model $\Lambda$ is attributed in part to dark matter and dark energy. The latest Plank mission measurements indicate that the universe should have around $68 \%$ of this dark energy, $5 \%$ ordinary matter and $27 \%$ dark matter. However, in 5DU, the question of the cosmological constant, $\Lambda$ should be examined differently. There is good possibility that $\Lambda$ now relates to the outward pressure created by the underlying branes and is manifest in the Hubble's constant. The aftermath outward pressure could be an alternative to dark energy and dark matter. The outward profile of the aftermath may or may not be uniform, which means there might be somewhat greater outward pressure in some parts of the universe and lesser in other parts. Though, we cannot be sure and lack such evidence, it is possible that the dark energy pockets identified in DES and the variations in $\mathrm{CMB}$ relate to the aftermath undulations.

\section{Universe of Multiple Branes}

The discussion above has indicated that we do not have a strong reason in support of a $4 \mathrm{D}$ big bang, which is widely accepted model of the evolution of the universe today. We do not at the moment refute $4 \mathrm{D}$ hypothesis entirely but there is an equal possibility of a 5D universe. Based on the Theorem of the Dimensionality of the Expanding Universe, we can at least conclude that we have an additional dimension to the expanding space-time of the universe. Figure 6 presents a visualization of a $5 \mathrm{D}$ big bang with $4 \mathrm{D}$ expanding branes.

While none of us have any knowledge of what happened at big bang and why it happened, the widely accepted assumption is of an explosion-inflation like ultra event some 14 billion years ago. However, if we look at the multibrane theory presented above, big bang may have continued in the aftermath. There remains a possibility that the process may have been sporadic with intermittent injection of energy or continuous injection of energy from a source of which we have no

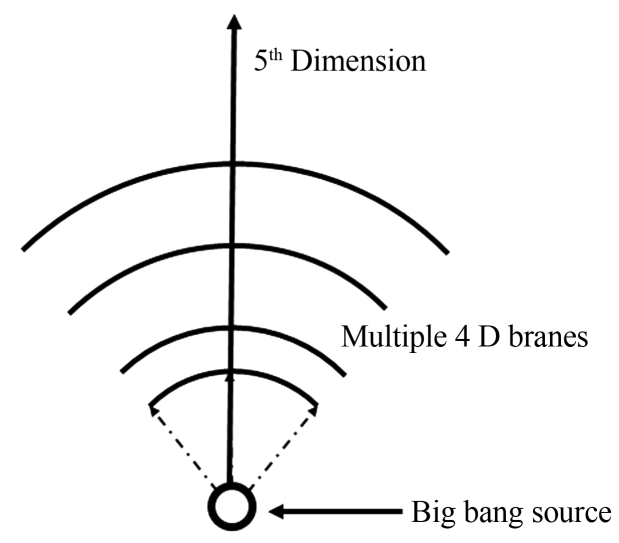

Figure 6.5D Big bang visualization. 
knowledge. This hypothesis appears more appealing than a one-time big-bang event that results in only our brane. Continuation of energy injection in the aftermath should also keep up the outward pressure to sustain the accelerated expansion of the universe. In that case dark matter and dark energy should be seen as manifestations of the multi-branes created in the aftermath. This is a better explanation of the outward isotropic force causing accelerated expansion than an unknown and undetectable dark matter and energy force.

\section{Discussion and Conclusions}

A review of mainly non-quantum aspects of the widely accepted Standard Model of Cosmology (SMC), 5D universe, and Extended Theories of Gravity (ETG) shows that many of the elements of SMC are a patchwork of different models or theoretical pieces that have been plugged-in in an attempt to explain different aspects of astrophysical observations such as CMB, red shift, Hubble's constant etc. However, these are standalone patchworks that do not necessarily emanate from a full end-to-end understanding of a process. Their purpose is limited to providing an explanation to the problem area or the gap in understanding. For example, let's take dark matter and dark energy, which have not been detected directly or the theory of inflation which explains the homogeneity in CMB but there seems to be no other reason for inflation and no process in GRT or quantum physics could be defined that may result in inflation and at the same time leads to other improbable scenarios such as any sub-atomic particle branching out as a separate universe. Similarly, we have no idea as to what dark matter and dark energy are and what is their genesis. Yet they form $95 \%$ of the matter and energy of the universe in SMC.

The CCC on the other hand presents an equivalence between the hot singularity and the cold expanded universe on account of high entropy and the mathematical basis of conformal geometry. However mathematics and physical realities can differ, and here we beg the question as to how one can treat a hot condensed state and an expanded cold state as equivalent. Apart from this there are many other lacunae in our understanding that we have highlighted above. We have the Hubble tension, lensing anomaly, baryon asymmetry, etc. and issues with the understanding of the cosmological constant. It is not difficult to conclude that there is something amiss and what has been theorized so far, known, and observed is not exactly what is out there.

The concept of a five dimensional universe (5DU) or a $(4+n)$ DU has been researched for almost 100 years but it is not a part of the SMC and one main reason for this has been a lack of any evidence or observation of a $5^{\text {th }}$ dimension. Different kinds of bulk and brane models have been proposed in which the brane is a thin membrane. The $(4+n)$ DU proponents, hypothesize $4 \mathrm{DU}$ as a very thin P-brane within a $(4+n)$ DU. In this approach some of the shortcomings of SMC seem to get resolved and there is a new notion for mass, which is related to the geometry in $(4+n)$ DU. Due to this reason and the many difficul- 
ties that we face in SMC, a $(4+n)$ DU or a 5DU appears to be more compelling model.

Our review and analysis with time dimension and the examination of the observed accelerated expansion of the universe and its dimensionality through a general case of a NDU leads us to some far reaching conclusions:

1) The theorem of the dimensionality of the expanding universe establishes that there should be another dimension to the expanding universe, which is not a part of the 4 dimensions (space-time).

2) Every object, microscopic, stellar or galactic has a spread or "thickness" in time

3) The observed $4 \mathrm{DU}$ is a thin undulating brane with a certain spread in the time dimension that varies with mass, scale and relative velocities.

The theorem necessitates the need for at least one more dimension beyond the 4 dimensions of space time. With these conclusions, one can re-examine bigbang as a $5 \mathrm{D}$ event and this point onwards there can be alternative hypotheses of how universe evolves from a 5D big bang. In this context, many questions arise-do we still need inflation or there is some other explanation possible? Similarly, the case of dark matter and dark energy-can there be a 5D construct that steps into the shoes of dark matter and dark energy?

Here we have proposed a new hypothesis of a $5 \mathrm{D}$ universe with multiple $4 \mathrm{D}$ branes that are generated by an injection of energy into the universe from an unknown source. The accelerated expansion of the universe is itself a possible signature of a $5 \mathrm{DU}$

We feel that it is unlikely that the big bang generated just one brane (our brane). What is more likely is that in addition to our brane, there may have been other branes that followed our brane in the aftermath. The branes in the aftermath could be exerting an outward pressure causing accelerated expansion of the universe and dark energy and dark matter could be manifestations of the underlying aftermath branes. For visualization and comparison sake, the analogy is with that of expanding and evolving nested hyper-involutes of 4D spheres with many layers to it. Undoubtedly, more research and fine mathematical work is needed to come to grips with this model of $4 \mathrm{D}$ expanding branes in a $5 \mathrm{DU}$ unleashed by a big bang and evolving as expanding nested hyper involutes of a $4 \mathrm{D}$ sphere.

Some interesting outcomes of the 5DU model are:

- Either we can do away with dark matter and dark energy or treat them as manifestations of the underlying aftermath branes.

- Possibilities of a large number (if not infinite) of universes (multiverse) as 4D branes in the aftermath.

- Contrary to the perceptions, big-bang may not have been a onetime event but if there are number of branes that follow our 4DU in the aftermath then it must have been a longer lasting event

- The total matter and energy content of the universe should be higher by 
many orders of magnitude.

- Lastly and most importantly, a 5D universe is structurally and physically very different from a $4 \mathrm{D}$ universe and arriving at a better understanding of it may require new physics.

In view of the many lacunae in SMC, as a way forward, this work recommends a greater focus in exploring 5DU with more fundamental work and to work towards detection of 5DU elements or signature or gravitational waves, as in ETG with advanced and more sensitive interferometers, and scientific programs designed for this purpose.

\section{Conflicts of Interest}

The author declares no conflicts of interest regarding the publication of this paper.

\section{References}

[1] Penrose, R. (1979) Singularities and Time-Assymetry. In: Hawking, S. and Israel, W., Eds., General Relativity, An Einstein Centenary Survey, Cambridge University Press, Cambridge, 581-638.

https://personal.lse.ac.uk/robert49/PPB/pdf/Penrose1979a.pdf

[2] Lincoln, D. (2021, January 5) Crisis in Cosmology Gets Worse. http://forbes.com https://www.forbes.com/sites/drdonlincoln/2021/01/05/crisis-in-cosmology-gets-w orse/?sh $=7 \mathrm{c} 9 \mathrm{~b} 49 \mathrm{~cd} 2826$

[3] Di Valentino, E., Mena, O., Pan, S., Visinelli, L., Yang, W., Melchiorri, A., et al. (2013) In the Realm of the Hubble Tension-A Review of Solutions. Classical and Quantum Gravity, 38, Article ID: 153001. arXiv:2103.01183v2 [astro-ph.CO] https://doi.org/10.1088/1361-6382/ac086d

[4] Sloman, L. (2019, July 29) Hubble Tension Headache: Clashing Measurements Make the Universe's Expansion a Lingering Mystery. Scientific American.

https://www.scientificamerican.com/article/hubble-tension-headache-clashing-mea surements-make-the-universes-expansion-a-lingering-mystery/

[5] Dainotti, M.G., De Simone, B., Schiavone, T., Montani, G., Rinaldi, E. and Lambiase, G. (2021) On the Hubble Constant Tension in the SNe Ia Pantheon Sample. The Astrophysical Journal, 912, 150. https://doi.org/10.3847/1538-4357/abeb73

[6] NASA (National Aeronautics and Space Administration) (2012, June 26) Galaxy Cluster and Giant Arc.

https://www.jpl.nasa.gov/images/galaxy-cluster-and-giant-arc

[7] News Staff (2021 June 16) 3.3-Billion-Light-Year-Long Arc of Galaxies Discovered. http://www.sci-news.com/astronomy/giant-arc-09768.html

[8] NASA (National Aeronautics and Space Administration) (2012, June 26) NASA's Hubble Spots Rare Gravitational Arc from Distant, Hefty Galaxy Cluster. https://hubblesite.org/contents/news-releases/2012/news-2012-19.html?news=true

[9] Stanford, S.A., Brodwin, M., Gonzalez, A.H., Zeimann, G., Stern, D., Dey, A., et al. (2011) IDCS 1426 + 3508: Discovery of A Massive, Ir-Selected Galaxy Cluster at $Z=$ 1.75. The Astrophysical Journal, 753, Article No. 164.

https://stsci-opo.org/STScI-01EVSRDN8F1DM6PC48XWQC7V6N.pdf https://doi.org/10.1088/0004-637X/753/2/164

[10] Riess, A.G., Filippenko, A.V., Challis, P., Clocchiatti, A., Diercks, A., Garnavich, 
P.M., et al. (1998) Observational Evidence from Supernovae for an Accelerating Universe and a Cosmological Constant. The Astronomical Journal, 116, Article No. 1009. https://doi.org/10.1086/300499

[11] Guth, A.H. and Kaiser, D.I. (2005) Inflationary Cosmology: Exploring the Universe from the Smallest to the Largest Scales. Science, 307, 884-890

https://doi.org/10.1126/science.1107483 https://www.science.org/doi/10.1126/science.1107483

[12] NASA (National Aeronautics and Space Administration) (n.d.) $\Lambda$ CDM Model of Cosmology. https://lambda.gsfc.nasa.gov/education/graphic history/univ evol.cfm

[13] Gohd, C. (2020, June 14) 'Standard Model' of Cosmology Called into Question by New Measurements.

https://www.space.com/universe-standard-model-hubble-constant-new-measureme nts.html

[14] Robson, B.A. (2019) Introductory Chapter: Standard Model of Cosmology. In: Robson, B.A., Ed., Redefining Standard Model Cosmology, IntechOpen, London, 1-4. http://dx.doi.org/10.5772/intechopen.85605

[15] Ade, P.A.R., Aghanim, N., Alves, M.I.R., Armitage-Caplan, C., Arnaud, M., Ashdown, M., et al. (2014) Planck 2013 Results. I. Overview of Products and Scientific Results. Astronomy \& Astrophysics, 571, Article No. A1. https://doi.org/10.1051/0004-6361/201321529

[16] Peebles, P.J.E. (1998) The Standard Cosmological Model. In: Greco, M., Ed., Principles of Physical Cosmology, Princeton University Press, Princeton, 3-10. https://doi.org/10.1515/9780691206721-003

[17] University of Cambridge (n.d.) David Tong: Lectures on Quantum Field Theory. http://www.damtp.cam.ac.uk/user/tong/qft.html

[18] Penrose, R. (2006) Before the Big Bang: An Outrageous New Perspective and Its Implications for Particle Physics. Proceedings of 10th European Particle Accelerator Conference (EPAC 2006), Edinburgh, 26-30 Jun 2006, 2759-2762.

https://accelconf.web.cern.ch/e06/papers/thespa01.pdf

[19] Randall, L. and Sundrum, R. (1999) A Large Mass Hierarchy from a Small Extra Dimension. Physical Review Letters, 83, 3370-3373.

https://doi.org/10.1103/PhysRevLett.83.3370

[20] Kaluza, Th. (2018) On the Unification Problem in Physics. International Journal of Modern Physics D, 27, Article ID: 1870001. https://doi.org/10.1142/S0218271818700017

[21] Wesson, P.S. (2012) Astronomy and the Fifth Dimension. The Observatory, 132, 372-376.

[22] Capozziello, S. and De Laurentis, M. (2011) Extended Theories of Gravity. Physics Reports, 509, 167-321. https://arxiv.org/abs/1108.6266 https://doi.org/10.1016/j.physrep.2011.09.003

[23] Harko, T., Lobo, F.S.N., Nojiri, S. and Odintsov, S.D. (2011) $f($ R, T) Gravity. Physical Review D, 84, Article ID: 024020. https://doi.org/10.1103/PhysRevD.84.024020

[24] Corda, C. (2009) Interferometric Detection of Gravitational Waves: The Definitive Test for General Relativity. International Journal of Modern Physics D, 18, 22752282. https://doi.org/10.1142/S0218271809015904

[25] Ryder, L. (2009) Introduction to General Relativity. Cambridge University Press, Cambridge. https://doi.org/10.1017/CBO9780511809033

[26] Einstein, A. (1905) Zur Elektrodynamik bewegter Körper. Annalen der Physik, 17, 891-921. https://doi.org/10.1002/andp.19053221004 
[27] Einstein, A. (1920) Relativity: The Special and General Theory. Lawson, R.W., Trans., Henry Holt \& Company, New York.

[28] Einstein, A. (1905) On the Electrodynamics of Moving Bodies. In: Cassidy, D.C. Renn, J. and Schulmann, R., Eds., The Swiss Years. Writings, 1900-1909 (English Translation Supplement) in the Collected Papers of Albert Einstein, Vol. 2, Princeton University Press, Princeton, 275-309. https://press.princeton.edu/books/hardcover/9780691085265/the-collected-papersof-albert-einstein-volume-2

[29] Einstein, A. (1915) The Field Equations of Gravitation. In: The Berlin Years. Writings, 1914-1917 (English Translation Supplement) in the Collected Papers of Albert Einstein, Vol. 6, Princeton University Press, Princeton, 117.

https://press.princeton.edu/books/paperback/9780691017341/the-collected-papersof-albert-einstein-volume-6-english

[30] Weinberg, S. (1989) The Cosmological Constant Problem. Reviews of Modern Physics, 61, 1-23. https://doi.org/10.1103/RevModPhys.61.1

[31] Carroll, S.M. (2004) Why Is the Universe Accelerating? In: Freedman, W.L., Ed., Carnegie Observatories Astrophysics Series, Measuring and Modeling the Universe (Vol. 2), Cambridge Univ. Press, Cambridge, 1-21.

[32] Rubin, D. and Hayden, B. (2016) Is the Expansion of the Universe Accelerating? All Signs Point to Yes. The Astrophysical Journal Letters, 833, Article No. L30. https://doi.org/10.3847/2041-8213/833/2/L30

[33] Bjorken, J.D. (2002, October) Cosmology and Standard Model. Stanford Linear Accelerator Center, Menlo Park. https://doi.org/10.1103/PhysRevD.67.043508 https://www.slac.stanford.edu/pubs/slacpubs/9250/slac-pub-9257.pdf

[34] Perlmutter, S., Aldering, G., Goldhaber, G., Knop, R.A., Nugent, P., Castro, P.G., et al. (1999) Measurements of $\Omega$ and $\Lambda$ from 42 High-Redshift Supernovae. The Astrophysical Journal, 517, 565-586. https://doi.org/10.1086/307221

[35] Akrami, J., Forveille, T., Pentericci, L. and Shore, S. (2019) Planck 2018 Results. I. Overview and the Cosmological Legacy of Planck. Astronomy \& Astrophysics, 641, Article No. E1, 56 p. https://doi.org/10.1051/0004-6361/202039265

[36] Smoot III, G.F. (2006, December 8) Cosmic Microwave Background Radiation Anisotropies: Their Discovery and Utilization. Nobel Lecture, 113-166. https://www.nobelprize.org/uploads/2018/06/smoot lecture.pdf

[37] Riess, A.G., Casertano, S., Yuan, W., Bradley Bowers, J., Macri, L., Zinn, J.C., et al. (2021) Cosmic Distances Calibrated to 1\% Precision with Gaia Edr3 Parallaxes and Hubble Space Telescope Photometry of 75 Milky Way Cepheids Confirm Tension with $\Lambda$ CDM. The Astrophysical Journal Letters, 908, Article ID: L6. https://doi.org/10.3847/2041-8213/abdbaf

[38] Di Valentino, E., Melchiorri, A. and Silk, J. (2020) Planck Evidence for a Closed Universe and a Possible Crisis for Cosmology. Nature Astronomy, 4, 196-203. https://doi.org/10.1038/s41550-019-0906-9

[39] Domenech, G., Chen, X., Kamionkowski, M. and Loeb, A. (2020) Planck Residuals Anomaly as a Fingerprint of Alternative Scenarios to Inflation. Journal of Cosmology and Astroparticle Physics, 10, Article No. 005.

https://doi.org/10.1088/1475-7516/2020/10/005 https://arxiv.org/pdf/2005.08998.pdf

[40] Ghosh, P. (2021) Dark Energy Survey Releases Most Precise Look at the Universe's Evolution. Science Correspondent.

https://www.bbc.com/news/science-environment-57244708 
https://news.fnal.gov/2021/05/dark-energy-survey-releases-most-precise-look-at-th e-universes-evolution/

[41] Blandford, R., Dunkley, J., Frenk, C., Lahav, O. and Shapley, A. (2020) Coming of Age of the Standard Model. Nature Astronomy, 4, 122-123.

https://doi.org/10.1038/s41550-020-1012-8 https://arxiv.org/ftp/arxiv/papers/2002/2002.12350.pdf

[42] Starr, M. (2021, June 18) A Ginormous Arc of Galaxies Was Just Detected in The Distant Universe.

https://www.sciencealert.com/there-appears-to-be-a-giant-arc-of-galaxies-in-the-di $\underline{\text { stant-universe }}$

[43] University of Central Lancashire (2021, June 7) Discovery of a Giant Arc in Distant Space Adds to Challenges to Basic Assumptions about the Universe.

https://www.uclan.ac.uk/news/discovery-of-a-giant-arc-in-distant-space-adds-to-ch allenges-to-basic-assumptions-about-the-universe

[44] Horváth, I., Bagoly, Z., Hakkila, J. and Tóth, L.V. (2015) New Data Support the Existence of the Hercules-Corona Borealis Great Wall. Astronomy \& Astrophysics, 584, Article No. A48. https://doi.org/10.1051/0004-6361/201424829

[45] Gunn, A. (n.d.) What Is the Largest Object in the Universe? https://www.sciencefocus.com/space/what-is-the-largest-object-in-the-universe/

[46] European Organization for Nuclear Research (CERN) (2021, March 23) LHCb Collaboration, Test of Lepton Universality in Beauty-Quark Decays. CERN-EP-2021042, LHCb-PAPER-2021-004.

[47] Canetti, L., Drewes, M. and Shaposhnikov, M. (2012) Matter and Antimatter in the Universe. New Journal of Physics, 14, Article ID: 095012. http://www.njp.org/ https://doi.org/10.1088/1367-2630/14/9/095012

[48] https://science.nasa.gov/astrophysics/focus-areas/what-is-dark-energy

[49] Choi, C.Q. (2017, June 17) Our Expanding Universe: Age, History \& Other Facts. https://www.space.com/52-the-expanding-universe-from-the-big-bang-to-today.ht $\underline{\mathrm{ml}}$

[50] https://astronomy.swin.edu.au/cosmos/b/big+bang

[51] Lemley, B. and Fink, L. (2020, April 1) Guth's Grand Guess. https://www.discovermagazine.com/the-sciences/guths-grand-guess

[52] https://wmap.gsfc.nasa.gov/universe/bb tests ele.html\#nuleosynthesis history

[53] https://www.darkenergysurvey.org/supporting-science/large-scale-structure/

[54] Penrose, R. (1965) Gravitational Collapse and Space-Time Singularities. Physical Review Letters, 14, 57-59. https://doi.org/10.1103/PhysRevLett.14.57

[55] Gao, C. (2012) A Model of Non-Singular Universe. Entropy, 14, 1296-1305. https://doi.org/10.3390/e14071296

[56] Malek, E. (2020) The Singularity Theorem (Nobel Prize in Physics 2020). https://www.einstein-online.info/en/spotlight/the-singularity-theorem/

[57] Hawking, S.W and Penrose, R. (1970) The Singularities of Gravitational Collapse and Cosmology. Proceedings of the Royal Society of London, Series A, 314, 529-548. https://doi.org/10.1098/rspa.1970.0021

[58] Nobel Lecture: Roger Penrose, Nobel Prize in Physics (2020) https://www.youtube.com/watch?v=DpPFn0qzYT0

[59] Ellis, J. (2012) The Need for New Physics. Nature, 481, 24. https://doi.org/10.1038/481024a 
[60] Garisto, D. (2021, April 7) Long-Awaited Muon Measurement Boosts Evidence for New Physics. Scientific American.

https://www.scientificamerican.com/article/long-awaited-muon-measurement-boos ts-evidence-for-new-physics/

[61] Hossain, J. (2019) Energy and Humanity: An Intertwined Evolution. Cambridge Scholars Publishing, Newcastle, UK.

[62] Science \& Cocktails (2019, August 27) String Theory and the End of Space and Time with Robbert Dijkgraaf. https://www.youtube.com/watch?v=0T--WC4D1C0

[63] Greene, B.R., Morrison, D.R. and Polchinski, J. (1998) String Theory. Proceedings of the National Academy of Sciences of the United States of America, 95, 1103911040. https://doi.org/10.1073/pnas.95.19.11039

[64] Tong, D. (2017, February 15) Quantum Fields: The Real Building Blocks of the Universe. https://www.youtube.com/watch?v=zNVQfWC evg

[65] Pease, R. (2001) Brane New World. Nature, 411, 986-988. https://doi.org/10.1038/35082684

[66] https://hubblesite.org/

[67] CERN (European Organization for Nuclear Research) (n.d.) Large Hadron Collider. https://home.cern/science/accelerators/large-hadron-collider

[68] https://www.nikhef.nl/ pkoppenb/particles.html

[69] https://www.ligo.caltech.edu/

[70] Hooft, G.T. (2010) Introduction to General Relativity. Institute for Theoretical Physics, Utrecht University, Utrecht.

[71] Grøn, Ø. and Hervik, S. (2004) Einstein's General Theory of Relativity. http://202.38.64.11/ jmy/documents/ebooks/Groen\%20O.,\%20Hervik\%20S.\%20Ein stein's\%20general\%20theory\%20of\%20relativity\%20(book\%20draft, \%202004)(538s) PGr.pdf

[72] Haradhan, M. (2013) Friedmann, Robertson-Walker (FRW) Models in Cosmology. Journal of Environmental Treatment Techniques, 1, 158-164.

[73] Carrol, S.M., Press, W.H. and Turner, E.L. (1992) The Cosmological Constant. Annual Review of Astronomy and Astrophysics, 30, 499-542. https://doi.org/10.1146/annurev.aa.30.090192.002435

[74] Carrol, S.M. (2001) The Cosmological Constant. Living Reviews in Relativity, 4, Article No. 1. https://doi.org/10.12942/lrr-2001-1

[75] Weyl, H. (1918) Gravitation and Electricity. Sitzungsber. Preuss. Akad, Berlin, 465480.

[76] Klien, O. (1926) The Atomicity of Electricity as a Quantum Theory Law. Nature, 118, 516. https://doi.org/10.1038/118516a0

[77] Lidsey, J.E., Romero, C., Tavakol, R. and Rippl, S. (1997) On Applications of Campbell's Embedding Theorem. Classical and Quantum Gravity, 14, 865-879. https://doi.org/10.1088/0264-9381/14/4/005

[78] Wesson, P.S. (2015) The Status of Modern Five-Dimensional Gravity: A Short Review: Why Physics Needs the Fifth Dimension. International Journal of Modern Physics D, 24, Article ID: 1530001. https://doi.org/10.1142/S0218271815300013

[79] Wesson, P.S. and de Leon, J.P. (1995) The Equation of Motion in Kaluza Klien Cosmology and its implications for Cosmology. Astronomy \& Astrophysics, 294, 1-7.

[80] Wesson, P.S. (2008) Mass and Machian General Relativity. arXiv:0811.2529. https://arxiv.org/abs/0811.2529 
[81] Wesson, P.S. and Overduin, J.M. (2013) Scaling Relations for the Cosmological "Constant" in Five-Dimensional Relativity. Advances in High Energy Physics, 2013, Article ID: 214172, 6 p. https://doi.org/10.1155/2013/214172

[82] Arkani-Hamed, N., Dimopoulos, S. and Dvali, G. (1998) The Hierarchy Problem and New Dimensions at a Millimeter. Physics Letters B, 429, 263-272. https://doi.org/10.1016/S0370-2693(98)00466-3

[83] Bhattacharya, G. (2017) Hierarchy Problem and BSM Physics. Pramana, 89, Article No. 53. https://doi.org/10.1007/s12043-017-1448-2

[84] Hawking, S. (2006) The Theory of Everything: The Origin and Fate of the Universe. Jaico Publishing House, Mumbai.

[85] Gribbin, J. (1993, August 20) Science: Could Time Run Backwards? New Scientist. https://www.newscientist.com/article/mg13918872-600-science-could-time-run-bac kwards/

[86] Skibba, R. (2020) Book Review: Crunch Rip, Freeze or Decay-How Will the Universe End. Nature, 584, Article No. 187. https://doi.org/10.1038/d41586-020-02338-w

[87] Davies, P.C.W. (1974) Second law of Thermodynamics. Nature, 248, 366. https://doi.org/10.1038/248366a0

[88] http://users.sussex.ac.uk/ waa22/relativity/What is gravitational time dilation.ht $\underline{\mathrm{ml}}$

[89] Maartens, R. and Koyama, K. (2010) Brane-World Gravity. Living Reviews in Relativity, 13, Article No. 5. https://doi.org/10.12942/lrr-2010-5

[90] Hubble, E. (1929) A Relation between Distance and Radial Velocity among ExtraGalactic Nebulae. Proceedings of the National Academy of Sciences of the United States of America, 15, 168-173. https://doi.org/10.1073/pnas.15.3.168

[91] Possel, M. (2010) Changing Places-Space and Time Inside a Black Hole. https://www.einstein-online.info/en/spotlight/changing_places/

\section{Notations and Abbreviations}

$\mathrm{N}$ dimensional universe is referred to as "NDU" and to a $\mathrm{N}$ dimensional hypersurface as "NDH"

Tensor notation: We have used both numerical and Greek symbols. The superscript is the dimension and subscript the coordinates 